\title{
Effects of Longitudinal Space Charge in Beams for Heavy-Ion Fusion
}

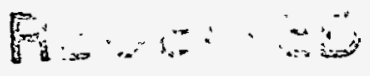 \\ FFP 0 O 1530 \\ W.M. Sharp, A. Friedman, and D.P. Grote \\ OSTI \\ Lawrence Livermore National Laboratory, University of California \\ P.O. Box 5508, L-440, Livermore, California 94550, USA
}

\begin{abstract}
This paper was prepared for submittal to the
Proceedings of the 8th ICFA Advanced Beam Dynamics Workshop on Space Charge Dominated

Beams and Applications of High Brightness Beams

Bloomington, Indiana, October 10-13, 1995
\end{abstract}

December 27, 1995

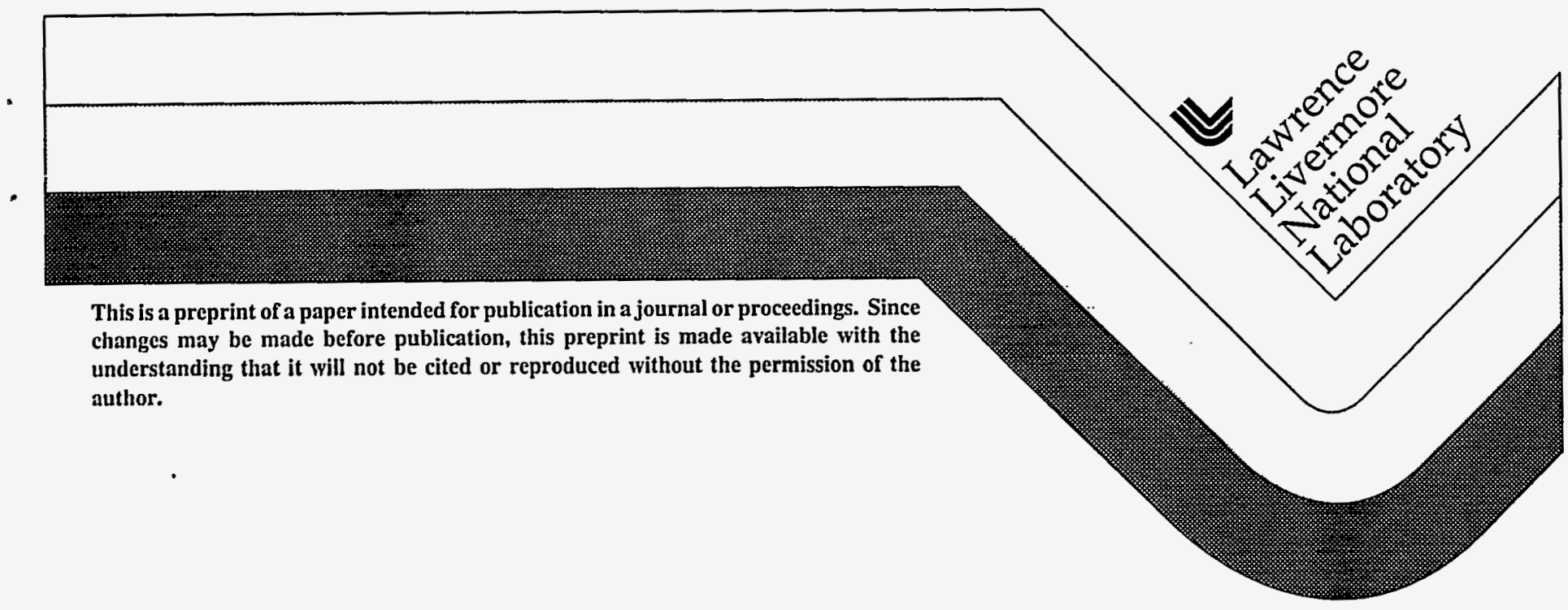

This is a preprint of a paper intended for publication in a journal or proceedings. Since ches may be made before publication, this preprint is made available with the understanding that it will not be cited or reproduced without the permission of the uthor. 
This document was prepared as an account of worksponsored by an agency of the United States Government. Neither the United States Government nor the University of Califormia nor any of their employees, makes any warranty, express or implied, or assumes any legal liability of responsibility for the accuracy, completeness, or usefulness of any information, apparatus, product, or process disclosed, or represents that its use would not infringe privately owned rights. Reference herein to any specific commercial products, process, or service by trade name, trademark, manufacturer, or otherwise, does not necessarily constitute or imply its endorsement, recommendation, or favoring by the United States Government or the University of California. The views and opinions of authors expressed herein do not necessarily state or reflect those of the United States Government or the University of California, and shall not be used for advertising or product endorsement purposes. 


\title{
EFFECTS OF LONGITUDINAL SPACE CHARGE IN BEAMS FOR HEAVY-ION FUSION*
}

\author{
W. M. Sharp, A. Friedman, and D. P. Grote \\ Lawrence Livermore National Laboratory L-440, Livermore, CA 94550, USA
}

\begin{abstract}
A new analytic model is presented that accurately estimates the radially averaged axial component of the space-charge field of an axisymmetric heavy-ion beam in a cylindrical beam pipe. The model recovers details of the field near the beam ends that are overlooked by simpler models, and the results compare well to exact solutions of Poisson's equation. Field values are shown for several simple beam profiles and are compared with values obtained from simpler models. The model has been implemented in the fluid/envelope code CIRCE and used to study longitudinal confinement in beams with a variety of axial profiles. The effects of errors in the longitudinal-control fields are presented.
\end{abstract}

\section{Introduction}

Longitudinal confinement of space-charge-dominated beams in induction accelerators requires detailed knowledge of the beam space-charge field. Unlike radio-frequency accelerators, the accelerating fields of induction accelerators provide no longitudinal focusing, so time-varying electric fields must be added to the acceleration field in at least some induction cells to balance the space-charge force.

For the ion beams considered for heavy-ion fusion (HIF), which are typically meters long and only a few centimeters in radius, the longitudinal space-charge field is a highly non-linear function of position in the beam frame, so the longitudinal-control fields, referred to here as "ears," must vary in time proportionally with the space-charge field as the beam moves through the acceleration gaps. The ideal ear field is related to the beam space-charge field at corresponding times by

$$
E_{\text {ear }} \approx-\eta_{\text {ear }}\left\langle E_{z}\right\rangle
$$

where the ear occupancy $\eta_{e a r}$ is the ratio of the induction-cell gap length to the distance between those cells in which ears are applied, termed "ear cells." The angle brackets on $\left\langle E_{z}\right\rangle$ denote a density-weighted average over the beam cross-section. This average is needed because the impulse an ion receives from an induction cell is approximately independent of the transverse location of the particle, making it impossible for the ear field to nullify the space charge at all points across the beam. Using ears calculated from the averaged space-charge field minimizes this inevitable error in longitudinal confinement.

In HIF experiments [1] and some analytic work [2], the beam space-charge field has been calculated from a simple one-dimensional model. For an axisymmetric beam with a uniform charge density $\rho$, it can be shown that neglecting the axial derivative in Poisson's equation leads to the simple result, in SI units, that

$$
\left\langle E_{z}(z)\right\rangle \approx-\frac{1}{4 \pi \epsilon_{0}}\left[\ln \left(\frac{R^{2}}{a^{2}}\right)\right] \frac{\partial \lambda}{\partial z} .
$$

* The research was performed under the auspices of the U. S. Department of Energy by Lawrence Livermore National Laboratory under Contract No. W-7405-ENG-48. 
Here, $\lambda=\pi \rho a^{2}$ is the beam line-charge density, and $z$ is axial distance in the beam frame. The logarithmic factor in Eq. (2) is called the "geometry factor" or "g-factor," and $a$ and $R$ in the term denote the radii of the beam and the beam pipe respectively. A slightly more general treatment, including the possible axial variation in $\rho$ and $a$, gives

$$
\left\langle E_{z}(z)\right\rangle \approx-\frac{1}{4 \pi \epsilon_{0}}\left\{\left[\frac{1}{2}+\ln \left(\frac{R^{2}}{a^{2}}\right)\right] \frac{\partial \lambda}{\partial z}-\frac{\lambda}{a} \frac{\partial a}{\partial z}\right\} .
$$

These simple expressions are not expected to be valid at the beam ends because neglecting the axial derivative in Poisson's equation is clearly invalid there. The failure of Eqs. (2) and (3) is evident, for example, for a beam with a uniform charge density. For this case, $a$ vanishes at the beam ends, and both $\left\langle E_{z}\right\rangle$ expressions unphysically become singular there.

In this paper, a Green's function is used to derive a more general expression for the radially averaged longitudinal space-charge field $\left\langle E_{z}\right\rangle$ of a nonrelativistic ion beam centered in a perfectly conducting cylindrical pipe. The expression is specialized to beams, like those in induction accelerators, that are much longer than their radius, and a closed-form approximation to $\left\langle E_{z}\right\rangle$ is obtained for the class of beams with $a / R \gtrsim 0.05$ at all points. This calculation is done in the beam frame, but since HIF beams are essentially nonrelativistic, $\left\langle E_{z}\right\rangle$ is effectively the same in the laboratory frame. The importance of beam-radius variation is illustrated by plotting the space-charge field for several beam profiles, and results of the new model are compared with predictions of the simpler $g$-factor models. Finally, effects of optimal and imperfect ear fields on beam dynamics are illustrated and discussed.

\section{Derivation}

A general expression for $\left\langle E_{z}\right\rangle$ is derived from a Green's function equivalent to that in Ref. [3]. The Green's function $G$ for the potential of a ring of charge with unit magnitude centered in a perfectly conducting pipe of radius $R$ is obtained from Poisson's equation, given in SI units for this case by

$$
\nabla^{2} G\left(r, z ; r^{\prime}, z^{\prime}\right)=\frac{1}{\epsilon_{0} r} \delta\left(r-r^{\prime}\right) \delta\left(z-z^{\prime}\right)
$$

where the primed coordinates'denote the source location, and unprimed coordinates are field points. A straightforward solution gives

$$
G\left(r, z ; r^{\prime}, z^{\prime}\right)=\frac{1}{\epsilon_{0} R} \sum_{n=1}^{\infty} \frac{J_{0}\left(\frac{\alpha_{n}}{R} r\right) J_{0}\left(\frac{\alpha_{n}}{R} r^{\prime}\right)}{\alpha_{n} J_{1}^{2}\left(\alpha_{n}\right)} \exp \left(-\frac{\alpha_{n}}{R}\left|z-z^{\prime}\right|\right),
$$

where $J_{0}$ and $J_{1}$ are Bessel functions of the first kind, and $\alpha_{n}$ denotes the $n$th zero of $J_{0}$. The potential $\phi$ for any axisymmetric charge distribution with density $\rho(r, z)$ is then found by integrating $G$ over all $r^{\prime}$ and $z^{\prime}$, and the corresponding longitudinal space-charge field is given by $E_{z}(r, z)=-\partial \phi(r, z) / \partial z$. When $\rho$ is assumed to be independent of $r$ within some radius $a(z)$, then the $E_{z}$ expression is trivially averaged over $r$, giving

$$
\left\langle E_{z}(z)\right\rangle=\frac{1}{\pi \epsilon_{0} R} \sum_{n=1}^{\infty} \frac{\left(\frac{2}{A_{n} a}\right) J_{1}\left(A_{n} a\right)}{\alpha_{n} J_{1}^{2}\left(\alpha_{n}\right)}\left[\int_{-L_{b} / 2}^{L_{b} / 2} d z^{\prime} \operatorname{sgn}\left(z-z^{\prime}\right) \frac{\lambda^{\prime}}{a^{\prime}} J_{1}\left(A_{n} a^{\prime}\right) \exp \left(-A_{n}\left|z-z^{\prime}\right|\right)\right] .
$$


Here, the notation $A_{n} \equiv \alpha_{n} / R$ has been introduced, and $z$ has been assumed to be zero at the beam midpoint, so that the ends of a beam of length $L_{b}$ are at $\pm L_{b} / 2$. Although the assumption of uniform charge density across a beam slice is never rigorously satisfied for real beams due to their nonzero transverse temperature, experiments [4] and particle simulations using space-charge-dominated beams show radial density profiles that are relatively flat near the axis and fall off rapidly near the edge. Such "top-hat" profiles are well approximated by a uniform density out to a radius chosen to give the measured or prescribed line-charge density $\lambda$.

The integral in Eq. (6) cannot in general be evaluated in closed form. However, for typical beams from induction accelerators, the axial scale lengths of $a$ and $\lambda$ are much longer than the exponential scale length $A_{n}^{-1}$ for all $n$. This short exponential scale length allows us to expand the $(\lambda / a) J_{1}$ factor linearly about the $z^{\prime}$ value where the integrand magnitude is maximum. Equating the derivative of the integrand with respect to $z^{\prime}$ to zero gives a transcendental equation for this integrand extremum. Rather than solve this equation numerically, we simplify the equation by assuming that $\lambda$ vanishes at the beam ends with a finite slope and by neglecting a term proportional to $\partial a / \partial z$, which is typically small for the long beams considered here. The resulting equation for the location of the integrand peak is

$$
1-\operatorname{sgn}\left(z-z^{\prime}\right) A_{n} \zeta^{\prime} \approx 0,
$$

where $\zeta(z)=\left(L_{b} / 2\right)-|z|$ is the axial distance from the nearest beam end. Examination of Eq. (7) shows that the location of the integrand peak is

$$
\zeta^{\prime} \approx \max \left(\zeta, A_{n}^{-1}\right) \equiv \zeta_{n}
$$

The significance of Eq. (8) is that the integrand is expanded about the exponential maximum except very near the ends.

After linear expansion of $(\lambda / a) J_{1}$ about $\zeta_{n}$, the integral in Eq. (6) is evaluated in a straightforward manner and, after some algebra, gives the following Bessel-series expression for $\left\langle E_{z}\right\rangle$ :

$$
\begin{array}{r}
\left\langle E_{z}(z)\right\rangle=\frac{1}{\pi \epsilon_{0}} \sum_{n=1}^{\infty} \frac{\left(\frac{2}{A_{n}^{\prime} a}\right) J_{1}\left(A_{n} a\right)}{\alpha_{n}^{2} J_{1}^{2}\left(\alpha_{n}\right)}\left\{\operatorname{sgn}(z) \frac{\lambda_{n}}{a_{n}} J_{1}\left(A_{n} a_{n}\right) \exp \left(-A_{n} \zeta\right)\right. \\
\left.-2\left[\frac{1}{A_{n} a} \frac{\partial \lambda}{\partial z} J_{1}\left(A_{n} a\right)-\frac{\lambda}{a} \frac{\partial a}{\partial z} J_{2}\left(A_{n} a\right)\right]_{\zeta_{n}} f_{n}(z)\right\},
\end{array}
$$

where $a_{n}$ and $\lambda_{n}$ are values at $\zeta_{n}$, and

$$
f_{n}(z) \equiv 1-\frac{1}{2}\left[1+\max \left(1, A_{n} \zeta\right)\right] \exp \left(-A_{n} \zeta\right)
$$

Here, the fact that $A_{n} L_{b} \gg 1$ has been used to discard exponentially small contributions from the farther of the two beam ends. This expression is found to be in excellent agreement with the exact expression Eq. (6) for every case examined.

Despite the assumption of axisymmetry, this model can be applied both to beams displaced from the accelerator axis and to beams in alternating-gradient (AG) lattices. 
Using a three-dimensional simulation code WARP3d [5], we find for $a / R=0.33$ that values of $\left\langle E_{z}\right\rangle$ for a centered beam and one displaced $0.14 R$ from the axis differ by no more than 2\%. Similarly, Eq. (9) can be applied to an elliptical beam by replacing the radius $a$ by the geometric mean $(a b)^{1 / 2}$ of the major and minor radii $a$ and $b$. For $a / b=1.8$, $\left\langle E_{z}\right\rangle$ differs from the field for the corresponding round beam by at most $4 \%$. Furthermore, since $a$ and $b$ fluctuate in the AG focusing on a period that is typically much smaller than the characteristic longitudinal-expansion time, the effect of beam ellipticity on longitudinal dynamics is substantially less than expected from this static comparison.

Eq. (9) is an important result of this paper, but the summation in general requires laborious numerical evaluation. In the following section, however, it is shown that the expression may be approximately evaluated for beams with sufficiently large radii at the beam ends.

\section{Special Cases}

\section{A. Beam Profiles}

The radius $a$ of the axisymmetric beam and the line-charge density $\lambda$ in Eq. (9) are in general independent quantities related by the beam transverse emittance and the accelerator lattice. In this work, $\lambda$ is taken to be any non-negative function that vanishes smoothly at the beam ends. For the equilibrium axisymmetric beams considered here, $a$, $\lambda$, and the normalized edge emittance $\epsilon_{N}$ are related approximately by the steady-state envelope equation

$$
-\frac{\sigma_{0}^{2}}{4 L^{2}} a+\frac{\kappa}{a}+\frac{\beta^{2} \epsilon_{N}^{2}}{a^{3}} \approx 0
$$

Here, $\sigma_{0}$ is the phase advance per lattice period $2 L$ in the absence of space-charge effects, and

$$
\kappa=\frac{1}{4 \pi \epsilon_{0}} \frac{2 e \lambda}{\beta^{2} M c^{2}} \equiv K \frac{\lambda}{\beta^{2}}
$$

is the beam perveance, with $\beta$ being the beam axial velocity scaled by $c$. Four cases are studied here:

(a) Uniform radius. Here, $\epsilon_{N}$ is obtained directly from Eq. (11) and increases toward the beam ends to balance the decreasing transverse space-charge force.

(b) Uniform normalized emittance. For this case, Eq. (11) is solved trivially for $a^{2}$, giving

$$
a^{2} \approx \frac{2 L^{2}}{\sigma_{0}^{2}}\left[K \frac{\lambda}{\beta^{2}}+\left(K^{2} \frac{\lambda^{2}}{\beta^{4}}+\frac{\beta^{2} \epsilon_{0}^{2} \sigma_{0}^{2}}{L^{2}}\right)^{1 / 2}\right] .
$$

(c) Uniform transverse temperature. The transverse temperature of a beam is, in an average sense, proportional to $T \equiv \epsilon_{N}^{2} / a^{2}$. If this temperature-like quantity is treated as uniform along a beam, Eq. (11) gives

$$
a^{2} \approx \frac{4 L^{2}}{\sigma_{0}^{2}}\left(K \frac{\lambda}{\beta^{2}}+T\right)
$$


(d) Uniform charge density. If the charge density $\rho$ is assumed uniform along the beam, then

$$
a^{2}=\frac{\lambda}{\pi \rho}
$$

and the normalized emittance $\epsilon_{N}$ from Eq. (11) vanishes at the beam ends along with $\lambda$ and $a$.

Although these simple profiles are unlikely to match that in an experimental beam, they illustrate the sensitivity of the space-charge field to the beam radial variation. Fig. 1 shows field values calculated for beams with identical parameters and line-charge profiles, but differing radial profiles. The parameters are those of a small recirculating induction accelerator [6] being developed at the Lawrence Livermore National Laboratory (LLNL), except that the midsection of the beam has been shortened to highlight field changes near the ends. As expected, one finds that the peak space-charge field increases for profiles that have smaller end radii. It is also evident that $\left\langle E_{z}\right\rangle$ for the uniform-density is qualitatively different from the others. For the cases with a finite beam-end radius, the field magnitude is seen to drop significantly in a narrow region at the beam end. In this region, which has a characteristic length of $R / \alpha_{1}$, the absence of charge outside the beam reduces the axial field, and at the endpoints, the field is reduced by approximately half. In contrast, $\left\langle E_{z}\right\rangle$ for the uniform-density case varies monotonically near the end due to the rapidly decreasing radius. Another distinction of the uniform-density case is that about 250 terms are required for convergence of the Bessel series in Eq. (9), whereas the other cases require between 20 and 40 terms. This difference arises because the beam radial profile is poorly fit by a Bessel series when $a / R$ is small, and many terms are needed for an adequate representation.

\section{B. Analytic Approximations}

When $a / R \gtrsim 0.05$ for all $z$, the sum in Eq. (9) converges rapidly and is dominated by the leading term. In this case, it is sensible to approximate the $\left\langle E_{z}\right\rangle$ expression by setting $n=1$ in $f_{n}$ and in the exponential factor. Also, since $a$ varies only slightly between the ends and $\zeta_{1}$ for such beams, leading Bessel factor and the derivatives of $a$ and $\lambda$ can all be evaluated at $\zeta_{1}$ with negligible error. These approximations leave two Bessel series that, remarkably, can be exactly summed. Expressed generally, these Bessel sums have been verified numerically over the range $1 \geq x>0$ :

$$
\begin{aligned}
& 4 \sum_{n=1}^{\infty} \frac{\left(\frac{2}{\alpha_{n} x}\right)^{2} J_{1}^{2}\left(\alpha_{n} x\right)}{\alpha_{n}^{2} J_{1}^{2}\left(\alpha_{n}\right)}=\frac{1}{2} \dot{+} \ln \left(\frac{1}{x^{2}}\right) \\
& 8 \sum_{n=1}^{\infty} \frac{\left(\frac{2}{\alpha_{n} x}\right) J_{1}\left(\alpha_{n} x\right) J_{2}\left(\alpha_{n} x\right)}{\alpha_{n}^{2} J_{1}^{2}\left(\alpha_{n}\right)}=1 .
\end{aligned}
$$

Outside the specified range, these sums either fail to converge or give other values. Substituting Eq. (16) into the approximate form of Eq. (9) leads to the expression

$$
\begin{aligned}
\left\langle E_{z}(z)\right\rangle=-\frac{1}{4 \pi \epsilon_{0}}\left\{[ \frac { 1 } { 2 } + \operatorname { l n } ( \frac { R ^ { 2 } } { a _ { 1 } ^ { 2 } } ) ] \left[\left.f_{1}(z) \frac{\partial \lambda}{\partial z}\right|_{\zeta_{1}}\right.\right. & \left.-\operatorname{sgn}(z) \frac{A_{1} \lambda_{1}}{2} \exp \left(-A_{1} \zeta\right)\right] \\
& \left.-f_{1}(z)\left[\frac{\lambda}{a} \frac{\partial a}{\partial z}\right]_{\zeta_{1}}\right\} .
\end{aligned}
$$




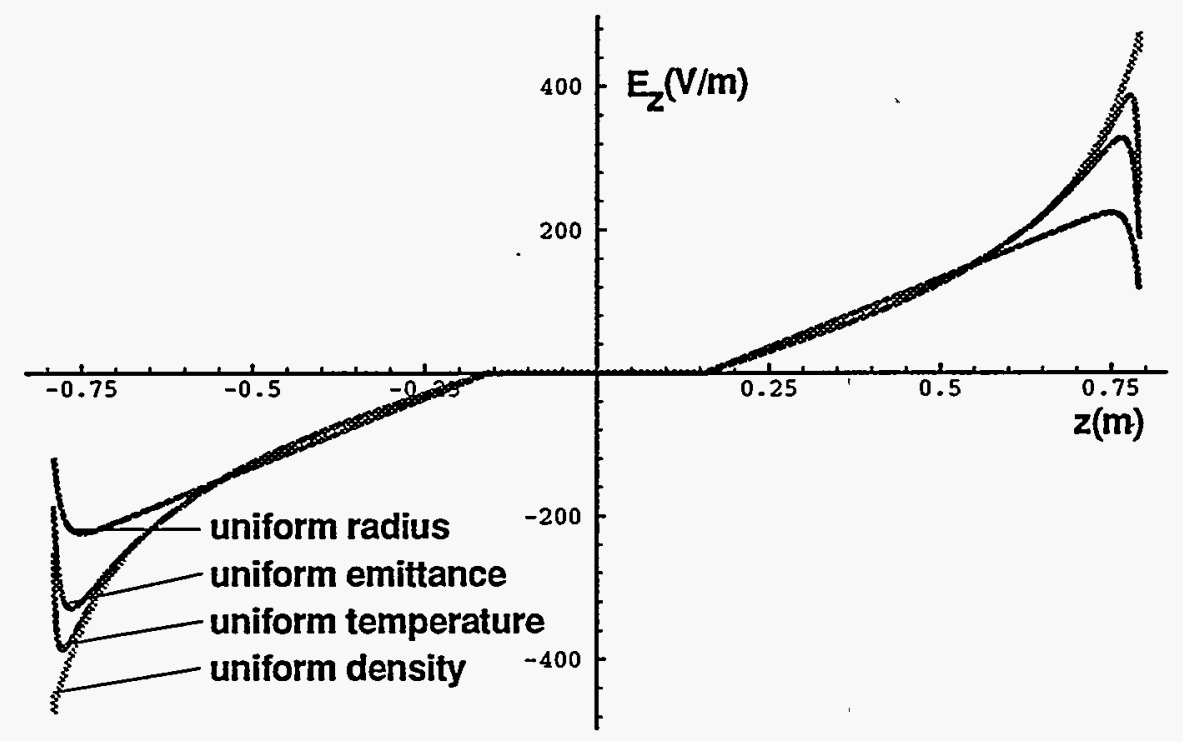

Fig. 1 Radially averaged space-charge field for beams with various radial profiles but the same line-charge density.

This expression is a numerically tractable generalization of Eq. (3) and is a very good approximation to the Bessel-series expression Eq. (9) for most experimental beams. As expected, it gives an inaccurate but non-singular result for uniform-density beams.

Sufficiently far from the beam ends, the exponentials in Eq. (9) vanish, and $\zeta_{n}=\zeta$. In this case, the Bessel sums in Eq. (16) can be used to evaluate Eq. (9) without further approximation, and the result exactly recovers the general g-factor expression Eq. (3). It follows that Eq. (3) gives $\left\langle E_{z}\right\rangle$ accurately in the interior of any beam in which $\lambda$ and $a$ vary on length scales that are long compared with $R / \alpha_{1}$. The expression only fails within a region a few times $R / \alpha_{1}$ in length at each end.

The various approximate expressions for $\left\langle E_{z}\right\rangle$ are compared in Fig. 2 for a beam with a uniform normalized emittance and the same lattice and beam parameters as the beams shown in Fig. 1. The Bessel-series approximation Eq. (9) has been compared with the exact integral expression Eq. (6) at selected points along the beam and is found to agree within $1 \%$ everywhere. The new analytic expression Eq. (17) deviates from the Bessel-series result by a few percent near the peak magnitude of $\left\langle E_{z}\right\rangle$, but it nonetheless reproduces the main features of the more exact expression. Both curves overlay the general $\mathrm{g}$-factor model away from the ends, as expected. The curve generated from the simple $g$-factor expression Eq. (2) deviates from the other approximations in the beam interior, underlining the fact that variation of the beam radius cannot in general be ignored.

\section{Effects on Dynamics}

\section{A. Calculation of Ears}

The fluid/envelope code CIRCE [7] is used here to assess the effects of space charge on the longitudinal dynamics of heavy-ion beams: Longitudinal dynamics is modeled in the code by treating slices of the beam as Lagrangian fluid elements. The boundaries between slices are assumed to be perpendicular to the beam axis and to respond to the average 


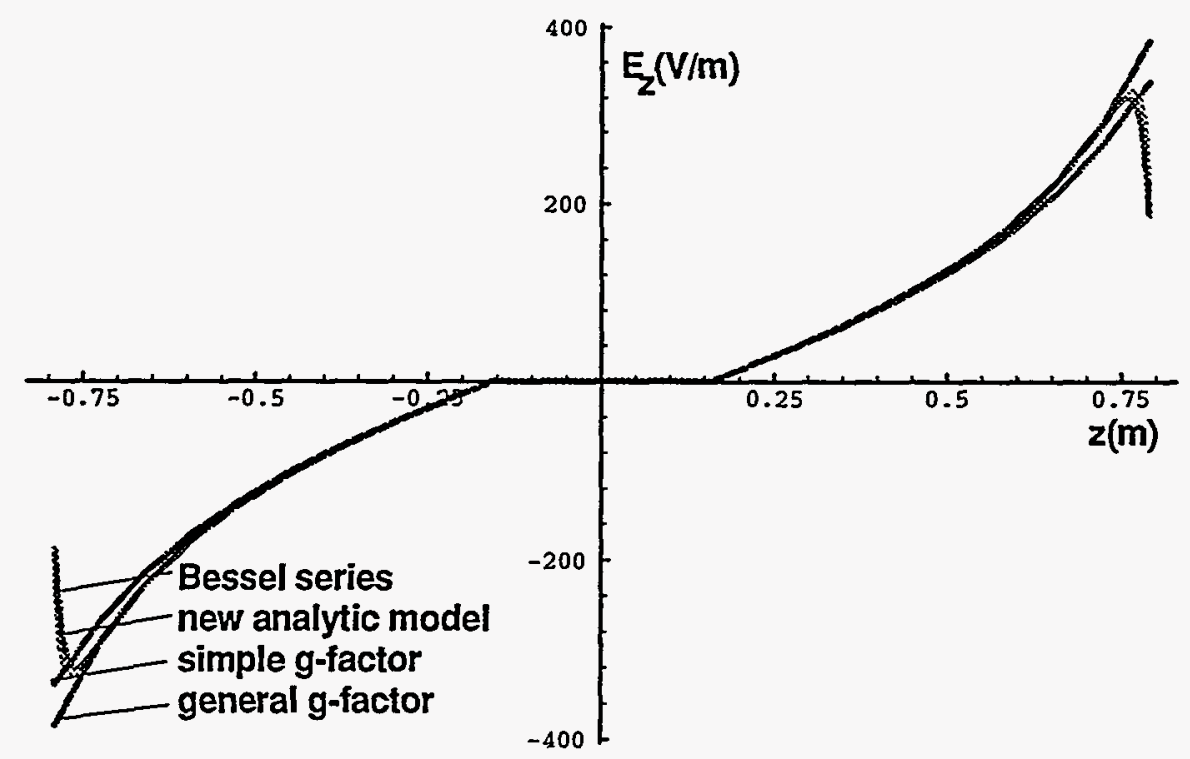

Fig. 2 Radially averaged space-charge field for a uniform-emittance beam calculated using various approximations

space-charge field $\left\langle E_{z}\right\rangle$ and to the axial field from acceleration cells. The neglect in this model of radial variation in the space-charge field is justified because this variation remains small so long as the space-charge potential across a beam is large compared with the transverse thermal energy, a condition that characterizes space-charge-dominated beams. The beam can be initialized using any of the emittance profiles discussed in section IIIA, and the electric field can optionally be calculated from the expressions in Eqs. (2), (3), (9), or (17).

Optimal ears are generated by a simple procedure. For a particular beam and lattice, CIRCE is run once without ear fields and with the axial space-charge force artificially switched off. This condition is equivalent to having perfect ears that are applied continuously. During the run, the average axial space-charge field $\left\langle E_{z}\right\rangle$ is calculated as the beam enters each ear cell, and Eq. (1) is used to obtain the corresponding ear fields, which are written along with timing data to an external file. Here, the general g-factor expression Eq. (3) is used to calculate $\left\langle E_{z}\right\rangle$ for beams with a finite emittance and radius at the ends, and the Bessel-series form Eq. (9) is used for beams with uniform charge density. Even though the g-factor expression misses the rapid field variation near the ends seen in Fig. 1, it is used here because the high frequency response needed to balance the space-charge field near the ends is beyond current pulse-generation technology. On subsequent runs, ears are obtained from the external file, and the space-charge field is calculated at every step from the Bessel series form Eq. (9) for constant density beams or otherwise from the new analytic expression Eq. (17).

\section{B. Dynamics with Optimal Ears}

The effects of optimal ears are illustrated here using the lattice and initial beam parameters of the small recirculating induction accelerator being developed at LLNL [6]. The initial current of singly charged potassium ions is $2 \mathrm{~mA}$, but the emittance is sufficiently low that at an initial energy of $80 \mathrm{keV}$, the beam transverse dynamics is space-charge dominated. In the uniform-emittance cases presented here, the beam doubles its energy 

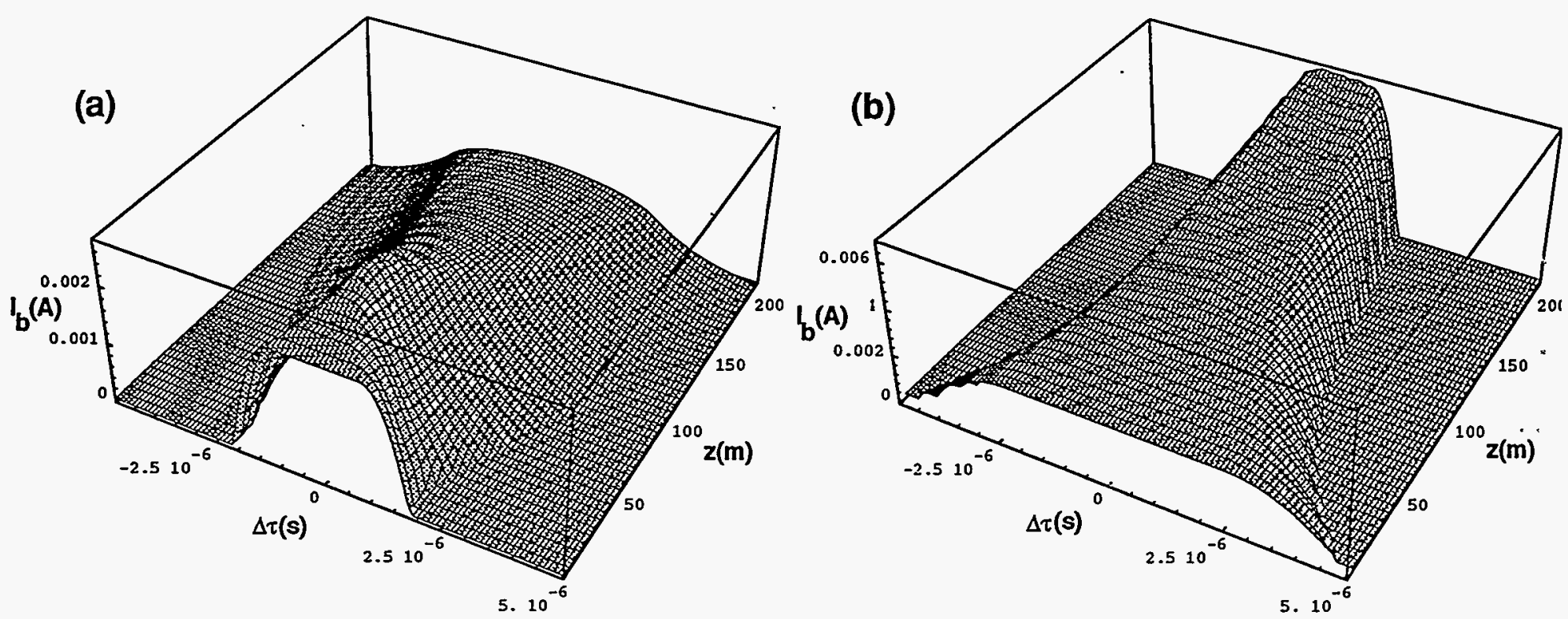

Fig. 3 CIRCE calculation of beam-current histories of a space-charge-dominated beam (a) without "ear" fields and (b) with space charge balanced by optimal ears applied periodically. Current is plotted as a function lattice position $z$ and time $\Delta \tau$ relative to the beam midpoint. Ripples seen in (b) near $z=0$ are a plotting artifact.

in 15 laps while compressing by a factor of four in duration and a factor of two in length. A head-to-tail velocity variation or "tilt" of about $35 \%$ is imposed during the first lap by triangular accelerating pulses in the first ten active cells, and ear fields are applied in each $36 \mathrm{~cm}$ half-lattice period (HLP), except in two sections of three HLPs each that are used for insertion and extraction of the beam.

In the absence of ears, space charge is seen in Fig. $3 a$ to push out the ends, and rarefaction waves move in from each end at the electrostatic wave speed $C_{s} \approx\left[g e q \lambda /\left(4 \pi \epsilon_{0} M\right)\right]^{1 / 2}$. The velocity tilt imposed by the first ten cells causes the density at the beam center to increase until, in the fourth lap; the rarefaction waves meet, and after that point the entire beam elongates to about 2.5 times the initial value. This case indicates that a beam can be transported without ear fields only over lattice lengths that are short compared with $\beta c L_{b} /\left(2 C_{s}\right)$.

With the axial component of the space-charge field set to zero, the beam current is found to compress in a nearly self-similar manner. "Using èirs generated by this first run, a second case, with space charge calculated from Eq. (17), gives the current history shown in Fig. 3b. On the scale shown, the pulse history is indistinguishable from that for the run without a longitudinal space-charge field, although more detailed plots show some low-amplitude space-charge waves launched during the first few laps. These waves result from the initial mismatch near the ends between the monotonically varying ear field and the rapidly varying space-charge field seen in Fig. 1 for a uniform emittance beam. Examination of the beam line-charge indicates, however, that the ends of the beam are quickly compressed, making the space-charge field approximately match the average ear field and effectively ending the generation of space-charge waves. A similar adaptation of the beam to the ear field is found with other beam emittance profiles. 

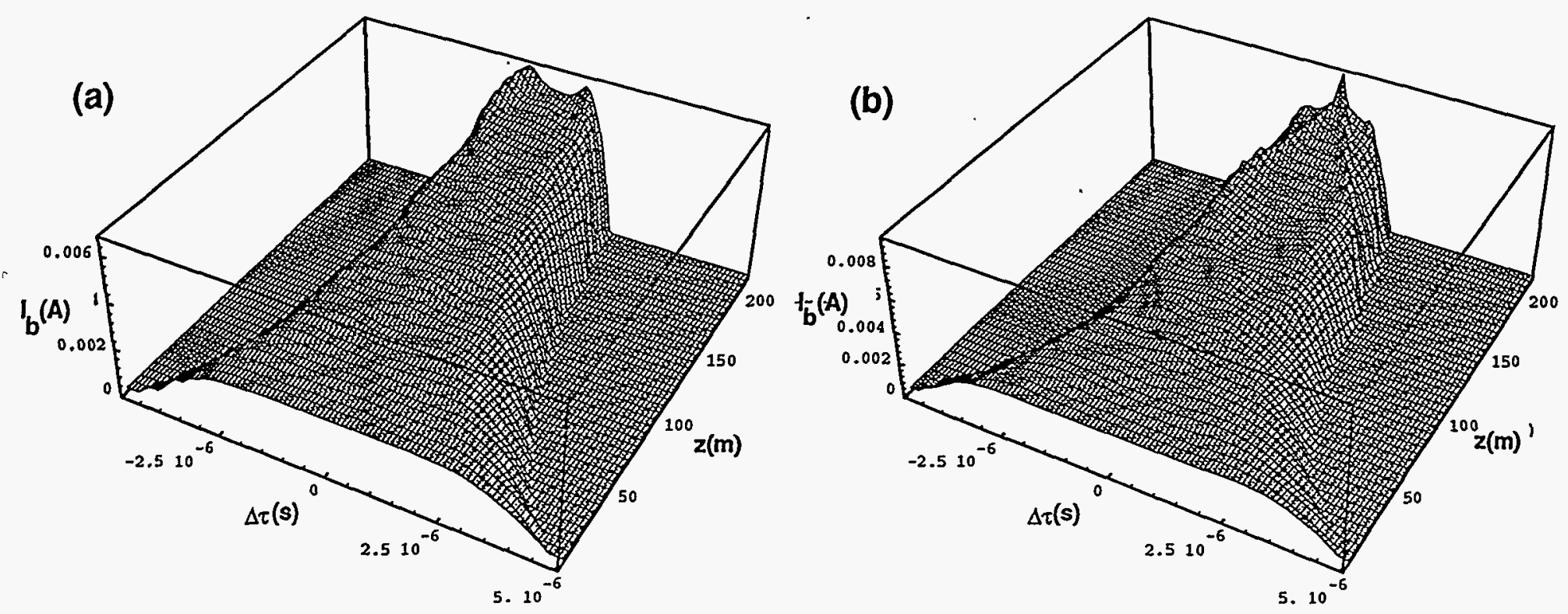

Fig. 4 CIRCE calculation of space-charge waves generated (a) by inappropriate current rise and fall times and (b) by an inappropriate current-profile form

If the optimal ears for a compressing beam are applied but no velocity tilt is imposed by the accelerating voltage, then the beam ends are pushed in according to the compression schedule, but large space-charge waves are generated as the ear fields move into the denser part of the beam. After six laps, the waves collide in a highly non-linear fashion that is not modeled correctly by CIRCE, illustrating that a velocity tilt is necessary for quiescent beam compression, as well as suitable ear fields.

Of necessity, the ear fields are applied periodically, so the ends are repeatedly being pushed in and then expanding. However, provided that this period is short compared with the characteristic time for beam expansion, these kicks are not an important source of noise. This consideration imposes a constraint on the maximum spacing of ear cells, but in practice, the breakdown voltage in induction cells is typically a more stringent limitation.

\section{Dynamics with Imperfect Ears}

Due to the imprecision in measuring the beam current profile and limitations in waveform generation, the ear fields used in an experiment are certain to be imperfect. A number of runs have been made to test qualitatively the sensitivity of beam dynamics to ear-field errors. For cases in which ear fields generated from one beam profile are applied to a beam with a different profile, the beam is found to be rather insensitive to the discrepancy for any pairing that involves the uniform emittance, density, or transverse temperature profiles in Eqs. (13)-(15). The reason for the insensitivity is that mismaiches between the average ear fields in these cases and the space-charge field become appreciable only in the low-density region at the ends, where $\lambda$ is less than about $10 \%$ of its mid-pulse value. In such cases, that beam is found to adapt to discrepancies as large as $60 \%$ between the ear and space-charge fields. Appreciable space-charge waves are generated, however, in beams with a uniform initial radius controlled by ears from one of the other profiles. As is evident from Fig. 1, the space charge for a uniform-radius beam differs visibly from that for the 
other profiles over almost half of the current rise and fall lengths, and the size of the field discrepancy is larger than in the previously cited cases. Both differences are expected to increase wave generation, but the effects have not been isolated and quantified.

A second series of runs was made using current profiles that differ from that used to generate the ear fields. Fig. $4 \mathrm{a}$ shows the current history of a beam with $1.2 \mu$ s current rise and fall times confined by ears calculated for a $1 \mu$ s rise and fall. In this case, the mismatch between the average ear field and the space-charge field occurs near the current flat-top, and sizable linear space-charge waves are generated. The waves are seen to increase in amplitude with $z$ because the fluid model used in CIRCE for longitudinal dynamics has no damping, so the wave energy is trapped in a progressively shorter length as the beam compresses. In an experiment or particle simulation, some damping is expected due to phase mixing and thermalization, and there would be a corresponding growth in longitudinal temperature. A more extreme mismatch is generated by specifying a cubic fall-off in the beam current near the ears but using ears calculated for a quadratic drop-off. The result, shown in Fig. $4 \mathrm{~b}$, is that larger linear waves are initially generated and become nonlinear after 15 laps. Both cases in Fig. 4 indicate that the beam is moderately sensitive to errors in the ear field that occur in the higher-density regions of the beam.

\section{Conclusions}

The new analytic model presented in Eq. (17) provides an accurate and convenient means for calculating the longitudinal component of the space-charge field in HIF beams. The assumptions of a non-vanishing emittance and a beam length that is much longer than the beam-pipe radius are typically satisfied for beams in induction accelerators. The other assumption of a uniform density across the beam profile is well enough satisfied by spacecharge-dominated beams that good quantitative agreement with experimental beams is expected when measured quantities like the current and the average radius are used in the model.

Using the new space-charge model, CIRCE can now generate optimal ear fields and test the sensitivity of beams to ear-field and beam-profile error. Although CIRCE simulations overestimate the wave amplitude due to errors in the longitudinal confinement and ignore the resulting emittance growth, the code appears to be a reliable tool for testing acceleration and compression schedules and for generating ear fields for use both in particle-simulation codes and in experiments.

\section{References}

[1] T. J. Fessenden, D. L. Judd, D. Keefe. C. Kim, L. J. Laslett, L. Smith, and A. I. Warwick, AIP Conference Proceedings 152 from the 1985 Heavy-Ion Fusion Symposium, Washington, D. C., p. 145.

[2] E. P. Lee, Proceedings of the 1981 Linear Accelerator Conference, Los Alamos Report LA-9234-C, p. 263.

[3] C. K. Allen, N. Brown, and M. Reiser, Part. Accel. 45, 149 (1994).

[4] M. G. Tiefenback, "Space-Charge Limits on the Transport of Ion Beams in a Long Alternating Gradient System, PhD Thesis, Lawrence Berkeley Laboratory Report LBL-22465 (1986).

[5] A. Friedman, D. P. Grote, and I. Haber, Phys. Fluids B 4, 2203 (1992). 
[6] A. Friedman, J. J. Barnard, M. D. Cable, D. A. Callahan, F. J. Deadrick, S. Eylon, T. J. Fessenden, D. P. Grote, H. A. Hopkins, V. P. Karpenko, D. L. Judd, H. C. Kirbie, D. B. Longinotti, S. M. Lund, L. A. Nattrass, M. B. Nelson, M. A. Newton, T. C. Sangster, and W. M. Sharp, "Recirculating Induction Accelerators for Inertial Fusion: Prospects and Status" in these proceedings.

[7] W. M. Sharp, J. J. Barnard, D. P. Grote, S. M. Lund, and S. S. Yu, "Envelope Model of Beam Transport in ILSE" in AIP Conference Proceeding 297 (AIP Press, Woodbury, NY,1994), p. 540. 


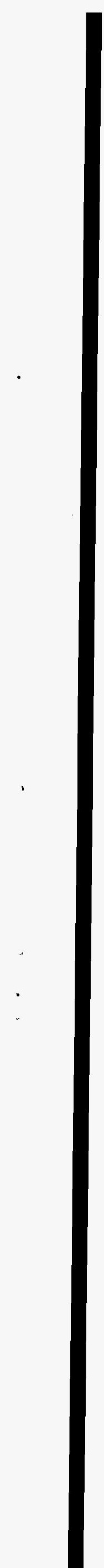


Techinical Information Departmemt . Lawrence Livermorc National Laboratory University of California - Livermorc, California 94551

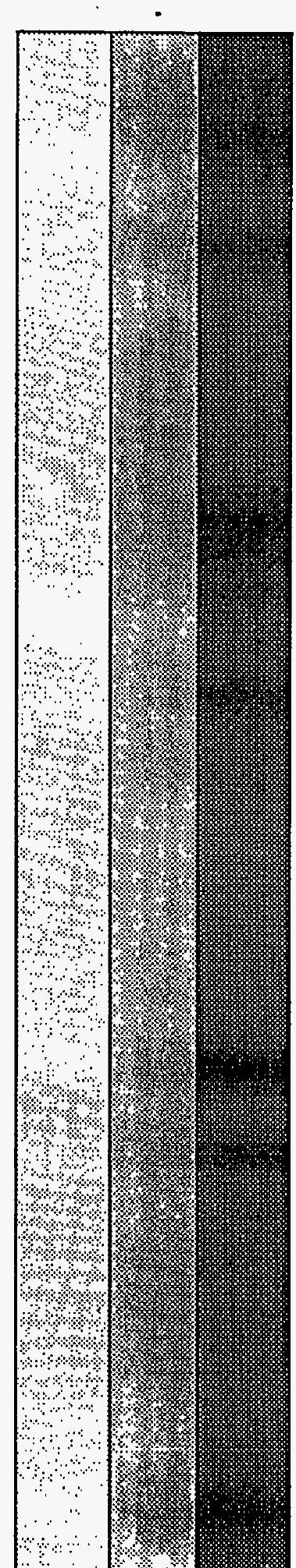

